\title{
Ample Vector Bundles on Open Algebraic Varieties
}

\author{
By \\ Shigeharu TAKAYAMA *
}

\section{§1. Introduction}

Let $X$ be a smooth projective variety over the complex number field. Let $D$ $=\sum_{l} D_{i}$ be an effective, reduced divisor on $X$ with only simple normal crossings, $D_{i}$ denoting its irreducible components. A vector bundle $E$ defined on $X$ is said to be ample modulo $D$, if for every torsion free sheaf $\mathscr{F}$ on $X$, there exists an integer $m_{0}>0$ such that $\left.\mathscr{F} \otimes S^{m}(E)\right|_{X-D}$ is generated by $H^{0}\left(X, \mathscr{F} \otimes S^{m}(E)\right)$ for $m \geq m_{0}$. A line bundle $L$ is very ample modulo $D$, by definition, if the rational map $\Phi_{|L|}$ attached to the linear system $|L|$ on $X$ gives an embedding of $X-D$ into some projective space.

There are many question in which we come across line bundles which are not ample but ample modulo $D$ for suitable $D$. For instans, let $X$ be a toroidal compactification of a locally symmetric space of rank one of non-compact type and take $D$ to be the boundary. In this case $K_{X}+D$ is nef and ample modulo $D$, but it is not ample in general (e. g. $X$ is a ball-quotient surface with ellipitic curves as the cusps).

The principle aim of the present article is to generalize a theorem of Demailly on ample line bundles (i. e. the case $D=0$ ) :

Theorem 1. There exists a function $C(n)$ in $n \in N$ with the following property: Let $X$ and $D$ be as above. Let $L$ be a nef line bundle on $X$ which is ample modulo $D$. Then $2\left(K_{X}+D\right)+m L$ is very ample modulo $D$ for any $m \geq C(\operatorname{dim} X)$.

In case $D=0$, this theorem was proved in [De 2] with aid of an analytic method such as Hörmander's $L^{2}$ estimates for the operator $\bar{\partial}$, Lelong number theory and Aubin-Yau's solution of the Calabi conjecture. Our proof of Theorem 1 proceeds in a similar way with necessary modifications.

Communicated by S. Mori, May 7, 1992.

1991 Mathematics Subject Classification : 32J25, 32L10, 14E-F.

* Department of Mathematics Tokyo Metropolitan University Minami-Ohsawa 1-1, Hachioji-shi Tokyo, 192-03 Japan. 
As a consequence of the proof of Theorem 1, we get

Theorem 2. Under the notation above, assume that $K_{X}+D\left(\right.$ resp. $\left.-\left(K_{X}+D\right)\right)$ is nef on an $n$-fold $X$ and ample modulo $D$. Then there exists a function $C(n)^{\prime}$ in $n \in N$ such that, if $m$ is greater than $C(n)^{\prime}, m\left(K_{X}+D\right)$ (resp. $\left.-m\left(K_{X}+D\right)\right)$ is very ample modulo $D$.

Theorem 2 is essential for the construction of the moduli space of open algebraic varieties of general type. Indeed consider a smooth family of open varieties with nice boundaries. If the logarithmic canonical divisors are nef and ample modulo $D$, then $m$-th logarithmic pluricanonical system defines a simultaneous embedding of the open varieties in a fixed projective space, so that the moduli space of such varieties will be obtained as a quotient of some Hilbert scheme.

I would like to express my gratitude to Professor Hajime Tsuji for suggesting to me this field of reseach, as well as for encouragement and many valuable discussions. I also would like to express my thanks to the referee for many nice advices.

\section{$\S$ 2. Criteria for Ampleness}

In this section, we give some criteria for a vector bundle to be ample modulo $D$, a divisor with simple normal crossings.

Proposition 2.1 ([Ha 1$]$ ). Consider the following two conditions on a vector bundle $E$ on $Y$, a scheme of finite type over an algebraically closed field $k$ :

(i) For every coherent sheaf $\mathscr{F}$ on $Y$, there is an integer $m_{0}>0$ such that for $m$ $\geq m_{0}$ and $i>0$

$$
H^{i}\left(Y, \mathscr{F} \otimes S^{m}(E)\right)=0
$$

(ii) $E$ is ample.

Then (2.1.1) (i) implies (ii).

(2. 1. 2) When $Y$ is proper, (ii) implies (i).

(2.1.3) Let $Y$ be proper, and let

$$
0 \longrightarrow E_{1} \longrightarrow E_{2} \longrightarrow E_{3} \longrightarrow 0
$$

be a short exact sequence of vector bundles on $Y$, with $E_{1}$ and $E_{3}$ ample. Then $E_{2}$ is ample. 
In general, if $Y$ is not proper over $k$, (ii) does not imply (i) and it is unknown whether (2.1.3) remains true ([Ha 1$])$ ). If we prove the coverse of (2. 1. 2) for our situation $Y=X-D$, an analogous assertion to (2.1.3) will follow (Corollary 2. 10 below). The proofs are based on vanishing theorems on complete Kähler manifolds.

For this, we shall review some analytic tools. Let $X$ be an $n$-dimensional complex projective manifold and let $L$ be a holomorphic line bundle over $X$ with a hermitian metric $h$. we denote the Chern curvature form of $h$ by $c(L)=c(L$, $h)=\frac{\sqrt{-1}}{2 \pi} \partial \partial \log h$, which is a $d$-closed real $(1,1)$-form representing the first Chern class $c_{1}(L) \in H^{2}(X, Z)$. It is well known than $L$ is ample if and only if $L$ has a smooth hermitian metric such that $c(L)$ is positive definite at every point. We also use singular metrics. By definition, a singular metric on $L$ is a metric which is given in any trivialization $\tau:\left.L\right|_{U} \cong U \times C$ by $\|\xi\|=|\tau(\xi)| e^{-\varphi(x)}$ for $x \in U, \xi \in L_{x}$, where $\varphi \in L_{l o c}^{1}(U)$ is a weight function. Then the curvature of $L$ is given by the $(1,1)$-current $c(L)=\frac{\sqrt{-1}}{\pi} \partial \bar{\partial} \varphi$ on $U$. For example, to any divisor $A=\sum \lambda_{i} A_{i}$ with coefficients $\lambda_{l} \in Z$ is associated with the invertible sheaf $\mathcal{O}$ ( $A$ ) of meromorphic functions $f$ such that $\operatorname{div}(f)+A \geq 0$; we can provide the bundle with the singular metric defind by $\|f\|=|f|$. If $g_{i}$ is a generator of the ideal of $A_{i}$ on an open set $U \subset X$ then $\tau(f)=f \prod g_{i}^{\lambda_{i}}$ defines a trivialization of $\mathcal{O}$ $(A)$ over $U$, and thus our singular metric has the weight function $\varphi=\sum \lambda_{i} \log$ $\left|g_{\imath}\right|$. By the Lelong-Poincaré equation (cf. $[\mathrm{G}-\mathrm{H}]$ ), we find

$$
c(\mathcal{O}(A))=\frac{\sqrt{-1}}{\pi} \partial \bar{\partial} \varphi=[A],
$$

where $[A]=\sum \lambda_{i}\left[A_{i}\right]$ denotes the current of integration over $A$. In what follows, a singular metric for the line bundle attached to an effective divisor is supposed to have positive curvature in the sence of current (cf. [Le]), i. e. the weight function $\varphi$ is plurisubharmonic.

Consider the real Neron-Severi spaces $N S_{R}(X)=\left(H^{2}(\mathrm{X}, \mathbb{Z}) \cap H^{1,1}(X)\right) \otimes R$ of algebraic cohomology classes of degree 2, and let $\Gamma_{+} \subset N S_{R}(X)$ (resp. $\Gamma_{a} \subset$ $\Gamma_{+}$) be the closed convex cone generated by cohomology classes of effective (resp. ample) divisors $D$; denote by $\Gamma_{+}^{\circ}\left(\operatorname{resp} . \Gamma_{a}^{\circ}\right)$ the interior of $\Gamma_{+}\left(\operatorname{resp} . \Gamma_{a}\right)$. Then, if $\omega$ is a Kähler metric on $X$ and $\varepsilon>0$, we have the following equivalences :

$c_{1}(L) \in \Gamma_{+} \Leftrightarrow L$ has a singular metric with $c(L) \geq 0$,

$c_{1}(L) \in \Gamma_{+}^{\circ} \Leftrightarrow \exists \varepsilon, L$ has a singular metric with $c(L) \geq \varepsilon \omega \Leftrightarrow \kappa(L)=n$,

$c_{1}(L) \in \Gamma_{a} \Leftrightarrow \forall \varepsilon, L$ has a smooth metric with $c(L) \geq-\varepsilon \omega \Leftrightarrow L$ is nef,

$c_{1}(L) \in \Gamma_{a}^{\circ} \Leftrightarrow \exists \varepsilon, L$ has a smooth metric with $c(L) \geq \varepsilon \omega \Leftrightarrow L$ is ample.

The notation $\kappa(L)$ stands for the Kodaira dimension of $L([\mathrm{Ii}])$. The following vanishing theorem is a key lemma for citeria for ampleness.

Proposition 2.3 (Hörmander's $L^{2}$ vanishing theorem, [Hö], [De 1], [Oh]). 
Let $M$ be a complex manifold with a complete Kähler metric $\omega, L$ be a line bundle on $M$ and let $E$ be a vector bundle on $M$. Assume that $L$ is equipped with a singular hermitian metric such that $c(L) \geq \varepsilon \omega$ for some $\varepsilon>0$. Then the $\mathbb{L}^{2}$ cohomology groups $H_{(2)}^{q}\left(M, \mathcal{O}\left(K_{M}\right) \otimes E \otimes L^{\otimes m}\right)=0$ for all large enough $m>0$ and $q>0$.

Let $X, D=\sum_{i} D_{i}$ be in $\S 1$ and let $E$ be a vector bundle on $X$. Let $F$ be an ample line bundle on $X$ with a hermitian metric $h_{F}$ of positive curvature. Then we can take

$$
\omega_{X-D}:=c\left(F, h_{F}\right)-\varepsilon_{0} \sqrt{-1} \sum_{i} \partial \partial \bar{\partial} \log \left(-\log \left\|\sigma_{i}\right\|_{i}^{2}\right)^{2}
$$

for $0<\varepsilon_{0} \ll 1$ as a complete Kähler metric on $X-D$, where $\sigma_{i}$ is a holomorphic section of $\mathcal{O}\left(D_{i}\right)$ which vanishes to first order on $D_{i}$, and $\left\|\sigma_{i}\right\|_{i}$ is the norm form a smooth hermitian metric on $\mathcal{O}\left(D_{i}\right)$ such that $\left\|\sigma_{i}\right\|_{i}<1$. Let $\mathscr{F}$ be a coherent sheaf on $X$. Since $X$ is projective, $\mathscr{F}$ can be written as a quotient of locally free sheaf (of finite rank) $\mathscr{L}$ on $X$. For any hermitian metric $h_{\mathscr{L}}$ on $\mathscr{L}$, we can construct a metric $h_{\mathscr{F}}$ on $\mathscr{F}$ associated with the following norm

$$
\|f\|:=\min \left\{\|g\|_{h_{\mathscr{L}}} ; g \in \mathscr{K} \text { such that the image of } g=f\right\} \text { for } f \in \mathscr{F} \text {. }
$$

With these symbols in mind, we have

Proposition 2.6. Assume the following condition:

(iii) For every torsion free sheaf $\mathscr{F}$ on $X$, there exists a hermitian metric $h_{E}$ on $E$ and there exists an integer $m_{0}>0$ such that for $m \geq m_{0}$ and $i>0$.

$$
H_{(2)}^{i}\left(X-D, \mathscr{F} \otimes S^{m}(E)\right)=0 .
$$

Then $E$ is ample modulo $D$.

Proof. In order for $E$ to be ample modulo $D$ it is sufficient that for every torsion free sheaf $\mathscr{F}$, and for every closed point $x \in X-D$, there be an integer $m_{0}>0$ such that for every $m \geq m_{0}$ the global sections of the sheaf $\mathscr{F} \otimes S^{m}(E)$ generate its stalk at the point $x([\mathrm{Ha} 1$, Proposition 2.1$])$. If we apply (iii) to $\mathscr{F} \otimes I_{x}$, where $I_{x}$ is the sheaf of ideals of $x$

$$
H_{(2)}^{1}\left(X-D, \mathscr{F} \otimes I_{x} \otimes S^{m}(E)\right)=0
$$

for all large enough $m$. So we have surjection

$$
H_{(2)}^{0}\left(X-D, \mathscr{F} \otimes S^{m}(E)\right) \longrightarrow\left(\mathscr{F} \otimes S^{m}(E)\right)_{x} \longrightarrow 0 .
$$


Thanks to the $L^{2}$ estimate, holomorphic sections of $\mathscr{F} \otimes S^{m}(E)$ on $X-D$ extend to holomorphic sections on $X$. Now we are done.

Next we give a sufficient condition for (iii). Let $\pi_{E}: P(E) \longrightarrow X$ be the projective space bundle and its natural projection associated with $E$, and let $\mathcal{O}_{E}$ (1) be the tautological line bundle on it.

Proposition 2. 7. Let $X, D$ and $E$ be as above. Assume the condition: (iv) There exist a hermitian metric $h_{\mathcal{O}_{E}(1)}$ on $\mathcal{O}_{E}(1)$ and a positive constant $c$ such that,

$$
c\left(\mathcal{O}_{E}(1), h_{\mathcal{O}_{E}(1)}\right) \geq c \pi_{E}^{*} \omega_{X-D}
$$

Then (iii) in Proposition 2. 6 holds.

Proof. Given a torsion free sheaf $\mathscr{F}$ on $X$, we can write $\mathscr{F}$ as a quotient of locally free sheaf (of finite rank) $\mathscr{L}$ on $X$. Let $\mathscr{K}$ be the kernel,

$$
0 \longrightarrow \mathscr{K} \longrightarrow \mathscr{L} \longrightarrow \mathscr{F} \longrightarrow 0
$$

Then $\mathscr{K}$ is also torsion free. For any metric on $\mathscr{L}$, we use the induced metric on $\mathscr{F}$. as in (2.5) and on $\mathscr{K}$ as a subsheaf of $\mathscr{L}$. We get the following exact sequence

$$
\begin{aligned}
\cdots \longrightarrow & H_{(2)}^{i}\left(X-D, \mathscr{L} \otimes S^{m}(E)\right) \longrightarrow H_{(2)}^{i}\left(X-D, \mathscr{F} \otimes S^{m}(E)\right) \\
& \longrightarrow H_{(2)}^{i+1}\left(X-D, \mathscr{K} \otimes S^{m}(E)\right) \longrightarrow H_{(2)}^{i+1}\left(X-D, \mathscr{L} \otimes S^{m}(E)\right) \longrightarrow \cdots
\end{aligned}
$$

By desending induction on $i$, it is sufficient to show (iii) for every vector bundle $\mathscr{L}$ on $X$.

We can take

$$
\tilde{\omega}:=\pi_{E}^{*} \omega_{X-D}-c\left(K_{P(E) / X}, h_{P(E) / X}\right)
$$

as a complete Kähler metric on $\boldsymbol{P}(E)-\pi^{-1}(D)$ for appropriate hermitian metric $h_{P(E) / X}$ on $K_{P(E) / X}$, note that $\pi^{-1}(D)$ is also a divisor with only simple normal crossings on $\boldsymbol{P}(E)$. Since

$$
H_{(2)}^{p}\left(X-D, R^{q} \pi_{E *}\left(\pi_{E}^{*}(\mathscr{L}) \otimes \mathcal{O}_{E}(1)^{\otimes m}\right)\right)=\left\{\begin{array}{cl}
H_{(2)}^{p}\left(X-D, \mathscr{L} \otimes S^{m}(E)\right) & \text { for } q=0, \\
0 & \text { for } q>0,
\end{array}\right.
$$

the Leray spectral sequence 


$$
\begin{gathered}
E_{2}^{p q}=H_{(2)}^{p}\left(X-D, R^{q} \pi_{E *}\left(\pi_{E}^{*}(\mathscr{L}) \otimes \mathcal{O}_{E}(1)^{\otimes m}\right)\right) \\
\Rightarrow E^{p+q}=H_{(2)}^{p+q}\left(P(E)-\pi^{-1}(D), \pi_{E}^{*}(\mathscr{L}) \otimes \mathcal{O}_{E}(1)^{\otimes m}\right)
\end{gathered}
$$

induces an isomorphism

$$
H_{(2)}^{i}\left(X-D, \mathscr{L} \otimes S^{m}(E)\right) \cong H_{(2)}^{i}\left(P(E)-\pi^{-1}(D), \pi_{E}^{*}(\mathscr{L}) \otimes \mathcal{O}_{E}(1)^{\otimes m}\right)
$$

for every $m$. In general, consider the cohomology group

$$
H_{(2)}^{i}\left(\mathbb{P}(E)-\pi^{-1}(D), \quad \mathcal{O}\left(K_{P(E)}\right) \otimes \mathscr{M} \otimes \mathcal{O}_{E}(1)^{\otimes m}\right),
$$

where $\mathscr{M}$ is a locally free sheaf on $\boldsymbol{P}(E)$. Since there exists a positive constant $\eta_{1}$ such that

$$
c\left(\mathcal{O}_{E}(1), h_{\mathscr{O}^{(1)}}\right) \geq-\eta_{1} c\left(K_{P(E) / X}, h_{P(E) / X}\right),
$$

there is a positive constant $\eta_{2}$ such that

$$
c\left(\mathcal{O}_{E}(1), h_{\mathbb{E}^{(1)}}\right) \geq \eta_{2} \tilde{\omega} \text {. }
$$

In this situation, by Proposition 2. 3, we have

$$
H_{(2)}^{i}\left(\mathbb{P}(E)-\pi^{-1}(D), \quad \mathcal{O}\left(K_{P(E)}\right) \otimes \mathscr{M} \otimes \mathcal{O}_{E}(1)^{\otimes m}\right)=0,
$$

so that

$$
H_{(2)}^{i}\left(X-D, \mathscr{L} \otimes S^{m}(E)\right)=0
$$

for $i>0$ and for all large enough $m$. Thus the proof is complete.

Remark 2. 8. Proposition 2.7 means that the degeneration of ampleness of $E$ near the boundary $D$ is not too fast, then $E$ has a good cohomological property (iii) in Proposition 2. 6. I do not know whether the converses of Proposition 2. 6 and Proposition 2. 7 are true.

But we can easily see :

Proposition 2.9. Let $X, D, E, F$ and $\omega_{X-D}$ be as in Proposition 2.6 and let $L$ be a line bundle on $X$. Assume that $E$ is ample modulo $D$ and that $L$ is ample on $X$. Then there exist a hermitian metric $h$ on $\mathcal{O}_{E \otimes L}(1)$ and a positive constant $\eta$ such that 


$$
c\left(\mathcal{O}_{E \otimes L}(1), h\right) \geq \eta \pi_{E \otimes L}^{*} \omega_{X-D} .
$$

Corollary 2. 10. Let $X$ and $D$ be as above, and let

$$
0 \longrightarrow E_{1} \longrightarrow E_{2} \longrightarrow E_{3} \longrightarrow 0
$$

be a short exact sequence of vector bundles on $X$, with $E_{1}$ and $E_{3}$ ample modulo $D$. Then $E_{2} \otimes L$ is ample modulo $D$ for any ample line bundle $L$ on $X$.

Proof. By the same argument of the proof of [Ha 1, Proposition 2. 2], $E_{1}$ $\oplus E_{3}$ is ample modulo $D$. Hence for each torsion free sheaf $\mathscr{F}$ there is an integer $n_{0}>0$ such that for $n \geq n_{0}$ and $i>0$,

$$
H_{(2)}^{i}\left(X-D, \mathscr{F} \otimes S^{n}\left(\left(E_{1} \oplus E_{3}\right) \otimes L\right)\right)=0
$$

by Proposition 2. 6, 2. 7 and 2.9. In other words, for $p+q \geq n_{0}, p, q \geq 0$,

$$
H_{(2)}^{i}\left(X-D, \mathscr{F} \otimes S^{p}\left(E_{1} \otimes L\right) \otimes S^{q}\left(E_{3} \otimes L\right)\right)=0,
$$

since $S^{n}\left(\left(E_{1} \oplus E_{3}\right) \otimes L\right)=\sum_{p, q \geq 0}^{p+q=n} S^{p}\left(E_{1} \otimes L\right) \otimes S^{q}\left(E_{3} \otimes L\right)$, and cohomology commutes with direct sums. Now $S^{n}\left(E_{2} \otimes L\right)$ has a filtration whose successive quotients are $S^{p}\left(E_{1} \otimes L\right) \otimes S^{q}\left(E_{3} \otimes L\right)$ for $p+q=n$ and $p, q \geq 0$. Hence, using the exact sequence of cohomology and proceeding step by step up the filtration, we deduce that

$$
H_{(2)}^{i}\left(X-D, \mathscr{F} \otimes S^{m}\left(E_{2} \otimes L\right)\right)=0
$$

for $i>0$ and for all large enough $m$. By Proposition 2.6, $E_{2} \otimes L$ is ample modulo $D$.

Remark 2. 11. We can see from the proof above, in the situation of Corollary 2. 10, $E_{2} \otimes L$ is not only ample modulo $D$ but also satisfies the good cohomological condition (iii) in Proposition 2. 6.

\section{§ 3. Vanishing Theorems and Existence of Holomorphic Sections}

In this section, we recall some results in [De 2] for some criteria $K_{X}+L$ to be spanned, very ample and so on for a nef and big line bundle $L$ on a projective $n$-fold $X$. The reader is referred to [De 2] for detail. Throughout of this section, we let $X$ be an $n$-dimensional projective manifold.

Let $T$ be a $d$-closed positive $(p, p)$-current on $X$. Let $(U, z)$ be a local 
coordinate centered at $x \in X$. The Lelong number of $T$ at $x$ is defined to be

$$
\nu(T, x)=\lim _{r \rightarrow+0} \frac{1}{\left(\pi r^{2}\right)^{n-p}} T\left(\chi_{r}\left(\frac{\sqrt{-1}}{2} \partial \bar{\partial}|z|^{2}\right)^{n-p}\right)
$$

where $\chi_{r}$ is the characteristic function of $B(r):$ the ball of radius $r$ in $U \subset \mathbb{C}^{n}$. The Lelong number exists and finite for all $d$-closed positive $(p, p)$-current and it is independent of the choice of the local coordinate (cf. $[\mathrm{G}-\mathrm{H}]$ ). In the case where $T$ is a current of integration $[A]$ over an analytic subvariety $A$, the Lelong number $\nu([A], x)$ coincides with the multiplicity of $A$ at $x(\mathrm{cf} .[\mathrm{G}-\mathrm{H}])$. By a theorem of $\mathrm{Siu}([\mathrm{Si}])$,

$$
E_{c}(T):=\{x \in X \mid \nu(T, x) \geq c\}
$$

is a union of subvarieties of codimension at least $p$ for every positive number $c$. Moreover $T$ can be written as a convergent series of $d$-closed positive currents

$$
T=\sum_{k=1}^{+\infty} \lambda_{k}\left[\mathbb{Z}_{k}\right]+\mathbb{R}
$$

where $\left[\mathbb{Z}_{k}\right]$ is a current of integration over a subvariety $\mathbb{Z}_{k}$ of codimension $p$ and $R$ is a residual current with the property that $\operatorname{codim} E_{c}(R)>p$ for every $c>$ 0 . This decomposition is locally and globally unique : the sets $Z_{k}$ are precisely the $p$-codimensional components occurring in the sublevel sets $E_{c}(T)$, and $\lambda_{k}=$ $\min _{x \in Z_{k}} \nu(T, x)$ is the generic Lelong number of $T$ along $\mathbb{Z}_{k}$. Let $\varphi$ be a plurisubharmonic function on $X$. The Lelong number of $\varphi$ can also be defined by

$$
\nu(\varphi, x)=\lim _{z \rightarrow x} \inf _{z} \frac{\varphi(z)}{\log |z|}
$$

with respect to $(U, z)$ : a local coordinate centered at $x$. It is known that $\nu(\varphi$, $x)$ is equal to the Lelong number $\nu(T, x)$ of the associated positive $(1,1)-$ current $T=\frac{\sqrt{-1}}{\pi} \partial \bar{\partial} \varphi$. Accordingly, we set $E_{c}(\varphi)=E_{c}(T)$. We also use the concept of multiplier ideal sheaf of $\varphi([\mathrm{Na}])$ which is the ideal subsheaf $\mathscr{I}(\varphi) \subset$ $\mathcal{O}_{X}$ of geams of holomorphic functions $f$ such that $|f|^{2} e^{-2 \varphi}$ is integrable with respect to the Lebesgue measure in some local coordinate. The zero variety $V \mathscr{I}(\varphi)$ is thus the set of points in a neighborhood on which $e^{-2 \varphi}$ is not integrable. This zero variety is closely related to the Lelong sublevel sets $E_{c}(\varphi)$. Indeed, if $\nu(\varphi, x)=\gamma$, the convexity properties of plurisubharmonic functions show that

$$
\varphi(z) \leq \gamma \log |z|+O(1) \quad \text { in a neighborhood of } x,
$$


and hence there exists a constant $C>0$ such that $e^{-2 \varphi(\mathrm{z})} \geq C|z|^{-2 \gamma}$ in a neighborhood of $x$. We easily infer that

$$
\nu(\varphi, x) \geq n+s \Rightarrow \mathscr{I}(\varphi){ }_{x} \subset \mathscr{M}_{X, x}^{s+1}
$$

where $\mathscr{M}_{X, x}$ is the maximal ideal of $\mathcal{O}_{X, x}$. In the opposite direction, it is known that $\nu(\varphi, x)<1$ implies the integrablity of $e^{-2 \varphi}$ in a neighborhood of $x$, that is, $\mathscr{I}(\varphi)_{x}=\mathcal{O}_{x, x}$. In particular, the zero variety $V \mathscr{I}(\varphi)$ of $\mathscr{I}(\varphi)$ satisfies

$$
E_{n}(\varphi) \subset V \mathscr{I}(\varphi) \subset E_{1}(\varphi)
$$

With these results and symbols in mind, we have

Lemma 3.3 $([\mathrm{Na}])$. For any plurisubharmonic function $\varphi$ on $X$, the ideal sheaf $\mathscr{I}(\varphi)$ is a coherent sheaf of ideals over $X$.

Proposition 3.4([Na]). Let $\omega$ be a Kähler metric on $X$ and let $L$ be a big line bundle over $X$. Assume that $L$ has a singular metric of weight $\varphi$ such that $c(L) \geq \varepsilon \omega$ for some $\varepsilon>0$. Then $H^{q}\left(X, \mathcal{O}\left(K_{X}+L\right) \otimes \mathscr{I}(\varphi)\right)=0$ for all $q \geq 1$.

Proposition 3.4 can be seen as a generalization of the Kawamata-Viehweg vanishing theorem $([\mathrm{Ka}],[\mathrm{Vi}])$. As a corollary of this, we have the following important

Corollary 3.5 ([De 2, Corollary 4.6]). Let $L$ be a nef and big line bundle over $X$. Assume that $L$ has a singular metric of weight $\varphi$ such that $c(L) \geq 0$ and let $x_{1}, \cdots, x_{N}$ be isolated points in the zero variety $V \mathscr{I}(\varphi)$. Then for every $\varepsilon>0$, there is a surjective map

$$
H^{0}\left(X, K_{X}+L\right) \rightarrow \bigoplus_{1 \leq j \leq N} \mathcal{O}\left(K_{X}+L\right)_{x_{j}} \otimes\left(\mathcal{O}_{X} / \mathscr{I}((1-\varepsilon) \varphi)\right)_{x_{j}} .
$$

The above result can be applied to construct sections of given line bundle, provided we are able to produce singular metrics with logarithmic poles. For this, we use the following existence theorem of Yau for solutions of MongeAmpère equations $([\mathrm{Ya}])$.

Lemma 3. 6. Let $\omega$ be a Kähler metric on $X$. Then for any smooth volume form $f>0$ with $\int_{X} f=\int_{X} \omega^{n}$, there exists a Kähler metric $\tilde{\omega}$ in the same Kähler class as $\omega$ such that $\tilde{\omega}^{n}=f$.

We first suppose that $L$ is an ample line bundle over $X$ and that $L$ is 
equipped with a smooth metric of positive curvature. Then consider the Kähler metric $\omega=c(L)$. Any form $\tilde{\omega}$ in the same Kähler class of $\omega$ can be written as $\tilde{\omega}$ $=\omega+\frac{\sqrt{-1}}{\pi} \partial \bar{\partial} \phi$, i. e. is the curvature form of $L$ after multiplication of the original metric by a smooth weight function $e^{-\psi}$. By Lemma 3.6, the MongeAmpère equation

$$
\left(\omega+\frac{\sqrt{-1}}{\pi} \partial \bar{\partial} \phi\right)^{n}=f
$$

can be solved for $\phi$, whenever $f$ is a smooth $(n, n)$-form with $\int_{X} f=L^{n}$. In order to produce logarithmic pole at given points $x_{1}, \ldots, x_{N} \in X$, let $f$ converge to a Dirac measure at $x_{j}$, then $\tilde{\omega}$ will be shown to converge to a closed positive $(1,1)$-current with non zero Lelong number at $x_{j}$. Let $\left(z_{1}, \ldots, z_{n}\right)$ be local coordinates centered at $\boldsymbol{x}_{j}$, defined on some neighborhood $V_{j} \cong\left\{|\boldsymbol{z}|<\boldsymbol{R}_{j}\right\}$. Let $g_{j}=\left(g_{j, 1}, \ldots, g_{j, n}\right)$ be arbitrary holomorphic functions on $V_{j}$ suth that $g_{j}^{-1}(0)=$ $\left\{\boldsymbol{x}_{j}\right\}$ and

$$
\log \left|g_{j}\right|=\log \left(\sum_{1 \leq k \leq n}\left|g_{j, k}\right|^{2}\right)^{1 / 2}
$$

Then $\log \left|g_{j}\right|$ has an isolated logarithmic pole at $x_{j}$ and $\left(\frac{\sqrt{-1}}{\pi} \partial \bar{\partial} \log \left|g_{j}\right|\right)^{n}=$ $\rho_{j} \delta_{x_{j}}$, where $\rho_{j}$ is the degree of the covering map $g_{j}:\left(\mathbb{C}^{n}, x_{j}\right) \rightarrow\left(C^{n}, 0\right)$. Indeed $\partial \bar{\partial} \log \left|g_{j}\right|=g_{j}^{*} \partial \bar{\partial} \log |w|$ has $\operatorname{rank}(n-1)$ on $V_{j}-\left\{x_{j}\right\}$ and

$$
\begin{aligned}
\int_{\left|g_{j}(z)\right|<r}\left(\frac{\sqrt{-1}}{\pi} \partial \bar{\partial} \log \left|g_{j}\right|\right)^{n} & =\frac{1}{\left(2 \pi r^{2}\right)^{n}} \int_{\left|g_{j}(z)\right|<r} g_{j}^{*}\left(\partial \bar{\partial} \log |w|^{2}\right)^{n} \\
& =\frac{\rho_{j}}{\left(2 \pi r^{2}\right)^{n}} \int_{|w|<r}\left(\sqrt{-1} \partial \bar{\partial} \log |w|^{2}\right)^{n}=\rho_{j}
\end{aligned}
$$

for every $r>0$ small enough. Now, let $\chi: \mathbb{R} \rightarrow \boldsymbol{R}$ be a smooth convex increasing function such that $\chi(t)=t$ for $t \geq 0$ and $\chi(t)=-\frac{1}{2}$ for $t \leq-1$. We set

$$
\alpha_{j, \varepsilon}=\frac{\sqrt{-1}}{\pi} \partial \bar{\partial}\left(\chi\left(\log \frac{\left|g_{j}\right|}{\varepsilon}\right)\right) .
$$

Then $\alpha_{j, \varepsilon}$ is a smooth positive $(1,1)$-form and $\alpha_{j, \varepsilon}=\frac{\sqrt{-1}}{\pi} \partial \bar{\partial} \log \left|g_{j}\right|$ over the set of points $z \in V_{j}$ such that $\left|g_{j}(z)\right|>\varepsilon$. It follows that $\alpha_{j, \varepsilon}^{n}$ has a support in the compact set $\left|g_{j}(z)\right| \leq \varepsilon$ and Stokes' formula gives

$$
\int_{V_{j}} \alpha_{j, \varepsilon}^{n}=\int_{V_{j}}\left(\frac{\sqrt{-1}}{\pi} \partial \bar{\partial} \log \left|g_{j}\right|\right)^{n}=\rho_{j} .
$$

Hence $\alpha_{j, \varepsilon}^{n}$ weakly converges to the Dirac measure $\rho_{j} \delta_{x_{j}}$ as $\varepsilon$ tends to 0 . For all positive numbers $\tau_{j}>0$ such that $\sigma=\sum \rho_{j} \tau_{j}^{n}<L^{n}$, Lemma 3.6 gives a solution of the Monge-Ampère equation 


$$
\omega_{\varepsilon}^{n}=\sum_{1 \leq j \leq N} \tau_{j}^{n} \alpha_{j, \varepsilon}^{n}+\left(1-\frac{\sigma}{L^{n}}\right) \omega^{n}
$$

with $\omega_{\varepsilon}=\omega+\frac{\sqrt{-1}}{\pi} \partial \widehat{\partial} \psi_{\varepsilon}$, since the right-hand side of the first equation is positive and has the correct integral $L^{n}$ over $X$. The solution $\phi_{\varepsilon}$ is only determined up to a constant. If $\gamma$ is an arbitrary Kähler metric on $X$, we can normalize $\phi_{\varepsilon}$ in such a way that $\int_{X} \psi_{\varepsilon} \gamma^{n}=0$.

Lemma 3.8 ( $\left[\right.$ De 2, Lemma 6,6]). There is a sequence $\varepsilon_{\nu}$ converging to zero such that $\phi_{\varepsilon_{\nu}}$ has a limit $\phi$ in $L^{1}(X)$ and that the sequence of $(1,1)$-forms $\omega_{\varepsilon_{\nu}}$ weakly converges towards a closed positive $(1,1)$-current $T$. The cohomology class of $T$ is equal to $c_{1}(L)$ and $T=\omega+\frac{\sqrt{-1}}{\pi} \partial \partial \partial$.

Let $U \subset X$ be an open coordinate patch that $L$ is trivial on a neighborhood of $\bar{U}$, and let $e^{-h}$ be the weight representing the initial hermitian metric on $L \mid \bar{U}$. Then $\frac{\sqrt{-1}}{\pi} \partial \widehat{\partial} h=\omega$ and $\frac{\sqrt{-1}}{\pi} \partial \bar{\partial}\left(h+\phi_{\varepsilon}\right)=\omega_{\varepsilon}$, so the function $\varphi_{\varepsilon}=h+\phi_{\varepsilon}$ defines a plurisubharmonic weight on $\left.L\right|_{\bar{U}}$, as well as its limit $\varphi=h+\phi$. By the continuity of $G$, Green operator associated with $\gamma$, we also infer that the family $\left(\phi_{\varepsilon}\right)$ is bounded in $L^{1}(X)$. The usual properties of subharmonic functions then show that there is a uniform constant $C$ such that $\varphi_{\varepsilon} \leq C$ on $\bar{U}$. We use this and equation (3.7) to prove that the limit $\varphi$ has logarithmic poles at all points $x_{j} \in$ $U$, thanks to Bedford and Taylor's maximum principle for solutions of MongeAmpère equations ( $[\mathrm{B}-\mathrm{T} 1])$ :

Lemma 3.9. Let $u, v$ be smooth (or continuous) plurisubharmonic functions on $\bar{U}$, where $U$ is a bounded open set in $C^{n}$. If

$$
u_{\left.\right|_{\partial U}} \geq\left. v\right|_{\partial U} \text { and }(\sqrt{-1} \partial \bar{\partial} u)^{n} \leq(\sqrt{-1} \partial \bar{\partial} v)^{n} \text { on } U \text {, }
$$

then $u \geq v$ on $U$.

In the application of Lemma 3.9, we suppose that $U$ is a neighborhood of $x_{j}$ and take

$$
u=\tau_{j}\left(\chi\left(\log \frac{\left|g_{j}\right|}{\varepsilon}\right)+\log \varepsilon\right)+C_{1}, \quad v=\varphi_{\varepsilon}
$$

where $C_{1}$ is a large constant. Then for $\varepsilon>0$ small enough

$$
\begin{gathered}
\left.u\right|_{\partial U}=\tau_{j} \log \left|g_{j}\right|+C_{1},\left.\quad v\right|_{\partial U} \leq C_{1}, \\
\left(\frac{\sqrt{-1}}{\pi} \partial \bar{\partial} \nu\right)^{n}=\omega_{\varepsilon}^{n} \geq \tau \alpha_{j, \varepsilon}^{n}=\left(\frac{\sqrt{-1}}{\pi} \partial \bar{\partial} u\right)^{n} \text { on } U .
\end{gathered}
$$

For $C_{1}$ sufficiently large, we infer $u \geq v$ on $U$, hence 


$$
\varphi_{\varepsilon} \leq \tau_{j} \log \left(\left|g_{j}\right|+\varepsilon\right)+C_{2} \text { on } U \text {. }
$$

So we have :

Corollary 3.10. The plurisubharmonic weight $\varphi=h+\phi$ on $\left.\mathbb{L}\right|_{U}$ associated with the limit function $\phi=\lim \psi_{\varepsilon_{\nu}}$ satisfies $\frac{\sqrt{-1}}{\pi} \partial \bar{\partial} \varphi=T$. Moreover, $\phi$ has logarithmic poles at all points $x_{j} \in U$ and

$$
\varphi(z) \leq \tau_{j} \log \left|g_{j}(z)\right|+O(1) \quad \text { in a neighborhood of } x_{j} \text {. }
$$

Case of a nef and big line bundle. Let $L$ be a nef and big line bundle and let $A$ be a fixed ample line bundle with smooth curvature form $\gamma=c(A)>0$. As $m L+A$ is ample for any $m \geq 1$, by Lemma 3.6, there exists a smooth hermitian metric on $L$ depending on $m$, such that $\omega_{m}=c(L)_{m}+\frac{1}{m} c(A)>0$ and

$$
\omega_{m}^{n}=\frac{\left(L+\frac{1}{m} A\right)^{n}}{A^{n}} \gamma^{n}
$$

However, we can not a priori control the assympotitic behaviour of $\omega_{m}$ when $m$ tends to infinity, so we introduce the sequence of not necessarily positive $(1,1)-$ forms $\omega_{m}^{\prime}=c(L)_{1}+\frac{1}{m} c(A) \in\left\{\omega_{m}\right\}$, which is uniformly bounded in $C^{\infty}(X)$ and coverges to $c(\mathbb{L})_{1}$. Then we solve the Monge-Ampère equation

$$
\omega_{m, \varepsilon}^{n}=\sum_{1 \leq j \leq N} \tau_{j}^{n} \alpha_{j, \varepsilon}^{n}+\left(1-\frac{\sigma}{\left(\mathbb{L}+\frac{1}{m} A\right)^{n}}\right) \omega_{m}^{n}
$$

with $\omega_{m, \varepsilon}=\omega_{m}^{\prime}+\frac{\sqrt{-1}}{\pi} \partial \bar{\partial} \psi_{m, \varepsilon}$ and with some smooth function $\phi_{m, \varepsilon}$ such that $\int_{X} \psi_{m, \varepsilon} \gamma^{n}=0$, this is again possible by Yau's theorem 3. 6. If we suppose

$$
\sigma=\sum \rho_{j} \tau_{j}^{n}<\mathbb{L}^{n}<\left(L+\frac{1}{m} A\right)^{n}
$$

then we solve (3.11) for all $m$. Then also there exist a convergent subsequence $\lim _{\nu \rightarrow+\infty} \psi_{m_{\nu}, \varepsilon_{\nu}}=\phi$ in $\mathbb{L}^{1}(X)$ and a closed positive $(1,1)$-current $\mathbb{T}=$ $\lim \omega_{m_{\nu}, \varepsilon_{\nu}}=c(L)_{1}+\frac{\sqrt{-1}}{\pi} \partial \bar{\partial} \phi \in c_{1}(L)$ such that Corollary 3.10 is still valid. In this case, $h$ is taken to be the weight function corresponding to $c(L)_{1}$.

Let us assume (with the notations of Corollary 3.10) that each point $x_{j}$ is isolated in $E_{1}(\varphi)$. Then we conclude by (3.2) and Corollary 3.5 that there is a surjective map

$$
H^{0}\left(X, K_{X}+L\right) \rightarrow \bigoplus_{1 \leq j \leq N} \mathcal{O}\left(K_{X}+\mathbb{L}\right)_{x_{j}} \otimes\left(\mathcal{O}_{X} / \mathscr{I}((1-\varepsilon) \varphi)\right)_{x_{j}}
$$


Finding sufficient conditions ensuring that $x_{j}$ is isolated in $E_{1}(\varphi)=E_{1}(T)$ is done in the next section. The rest of this section, we explain how to choose the logarithmic poles $\log \left|g_{j}\right|$ and the contants $\tau_{j}$ to obtain specified ideals and jets of sections at each point $x_{j}$. Suppose that an ideal $\mathscr{J}_{j} \subset \mathscr{M}_{X, x_{j}}$ is given at point $x_{j}$, in other words, that we are given a 0 -dimensional subscheme $\left(E, \mathcal{O}_{E}\right)$ with $\Xi=\left\{x_{1}, \ldots, x_{N}\right\}$ and $\mathcal{O}_{E, x_{j}}=\mathcal{O}_{X, x_{j}} / \mathscr{J}_{J}$. We want to find sufficient conditions for the surjectivity of the restriction map

$$
H^{0}\left(X, K_{X}+L\right) \longrightarrow H^{0}\left(\Xi, \mathcal{O}_{\Xi}\left(K_{X}+L\right)\right)=\bigoplus_{1 \leq J \leq N} \mathcal{O}\left(K_{X}+L\right)_{x_{J}} \otimes \mathcal{O}_{X, x_{J}} / \mathscr{J}_{J} .
$$

By (3. 12), we need only find a germ of map $g_{j}:\left(X, x_{j}\right) \longrightarrow\left(C^{n}, 0\right)$ and a constant $\tau_{j, 0}$ such that $\mathscr{I}\left(\tau_{j, 0} \log \left|g_{j}\right|\right) \subset \mathscr{J}_{j}$. For $\tau_{j}>\tau_{j, 0}$ and $\varepsilon$ small enough, Corollary 3. 10 then implies $\mathscr{I}((1-\varepsilon) \varphi) \subset \mathscr{J}_{j}$. Thus we have to choose $\sigma$ slightly larger than $\sigma_{0}=\sum \rho_{j} \tau_{j, 0}^{n}$ where $\rho_{j}$ is the degree of the covering map $g_{j}$, this is possible only if $L^{n}>\sigma_{0}$. Let us discuss some specific cases.

Spannedness. To obtain that $K_{X}+L$ spans at $x \in X$, we consider a single point $x_{1}=x$ and take $\mathscr{J}_{1}=\mathscr{M}_{X, x}, g_{1}(z)=\left(z_{1}, \ldots, z_{n}\right), \tau_{1,0}=n$ and $\sigma_{0}=\tau_{1,0}^{n}=$ $n^{n}$. Then $\mathscr{I}\left(\tau_{1,0} \log \left|g_{1}\right|\right) \subset \mathscr{M}_{X, x}$ as desired.

Separation of points. To obtain the separation of two points $x_{1} \neq x_{2}$ in $X$ by sections of $K_{X}+L$, we make the same choices as above at $x_{1}, x_{2}$ and get $\sigma_{0}=\tau_{1,0}^{n}$ $+\tau_{2,0}^{n}=2 n^{n}$. If $x_{1}, x_{2}$ are infinitely near in some direction $\xi \in T X$, we choose coordinates $\left(z_{1}, \ldots, z_{n-1}, z_{n}^{2}\right)$. We can choose $g_{1}(z)=\left(z_{1}, \ldots, z_{n-1}, z_{n}^{2}\right)$ and $\tau_{1,0}=n$. Then the degree of $g_{1}$ is $\rho_{1}=2$ and we find again $\sigma_{0}=\rho_{1} \tau_{1,0}^{n}=2 n^{n}$.

Corollary 3.13. Let $L$ be $a$ nef and big line bundle. A sufficient condition for spannedness (resp. separation of points) of $K_{X}+L$ on a given set $\Xi$ is $L^{n}>\sigma_{0}$ with $\sigma_{0}=n^{n}$ (resp. $\sigma_{0}=2 n^{n}$ ) provided that the solution $\omega_{\varepsilon}$ of (3.7), resp. the solution $\omega_{m, \varepsilon}=\omega_{m}^{\prime}+\frac{\sqrt{-1}}{\pi} \partial \bar{\partial} \psi_{m, \varepsilon}$ of (3.11), always has a subsequence converging to a current $T$ for which all points $x \in \Xi \cap E_{1}(T)$ are isolated in $E_{1}(T)$.

\section{§ 4. Generalization of Demailly's Self-intersection Inequality for Closed Positive Current}

In this section, we generalize some results of Demailly ([De 2, Theorem 10. 7] and so on) with the aid of the criteria in Section 2 for a vector bundle to be ample modulo $D$. Through out of this section, we use the following notations

$X$ : an $n$-dimensional projective manifold,

$D$ : a divior on $X$ with simple normal crossings, and

$L$ : a nef line bundle on $X$.

Let $T=c(L) \geq 0$ be the curvature current of any singular metric on $L$. We want to derive a bound for the codimenion $p$ components in the sublevel sets 
$E_{c}(T)$ in terms of the $p$ th power $\{T\}^{p}$ of the cohomology class of $T$. In general, $T^{p}$ does not make sense as a current. However, products of currents can be defined in some special circumstances. Suppose that $\Theta$ is a closed positive $(p, p)$ -current and that $\phi$ is a locally bounded plurisubharmonic function on a complex manifold $M$. According to Bedford-Taylor ( $[\mathrm{B}-\mathrm{T} 2]$ ), the product $\Theta \wedge$ $\sqrt{-1} \partial \bar{\partial} \phi$ can be defined by

$$
\Theta \wedge \sqrt{-1} \partial \bar{\partial} \phi=\sqrt{-1} \partial \bar{\partial}(\phi \Theta)
$$

Here $\Theta$ is a differential form with measure coefficients, so its product by the locally bounded Borel function $\phi$ is a well defined current of order 0 , and the derivative $\partial \bar{\partial}$ can be taken in the sense of distribution theory. The resulting current $\Theta \wedge \sqrt{-1} \partial \bar{\partial} \phi$ is again positive, as is seen by taking the weak limit with a sequence of smooth approximations of $\phi$. More generally, if $\phi_{1}, \ldots, \phi_{m}$ are locally bounded plurisubharmonic functions, the product $\Theta \wedge \sqrt{-1} \partial \bar{\partial} \psi_{1} \wedge \ldots \wedge$ $\sqrt{-1} \partial \partial \partial \psi_{m}$ is well defined by induction on $m$. Various example (cf. [Ki]) show that such products can not be defined for arbitrary plurisubharmonic functions $\phi_{j}$. However, functions with $-\infty$ poles can be admitted if the polar set is sufficiently small.

Proposition 4.2 ([De 2, Proposition 10.2]). Let $\phi$ be a plurisubharmonic function on $M$ such that $\phi$ is locally bounded on $M-A$, where $A$ is an analytic subset of $M$ of codimension $\geq p+1$ at each point. Then $\Theta \wedge \sqrt{-1} \partial \bar{\partial} \phi$ can be defined in such $a$ way that $\Theta \wedge \sqrt{-1} \partial \bar{\partial} \psi=\lim _{\nu \rightarrow+\infty} \Theta \wedge \sqrt{-1} \partial \bar{\partial} \psi_{\nu}$ in the weak topology of currents, for any decreasing sequence $\left(\phi_{\nu}\right)_{\nu \geq 1}$ of plurisubharmonic functions converging to $\phi$. Moreover, at every point $x \in M$ we have

$$
\nu\left(\Theta \wedge \frac{\sqrt{-1}}{\pi} \partial \bar{\partial} \phi, x\right) \geq \nu(\Theta, x) \nu(\phi, x) .
$$

By the Lebesgue decomposition theorem, we can write $T=c(L)=T_{\mathrm{abc}}+$ $T_{\text {sing }}$ where $T_{\text {abc }}$ has absolutely continuous coefficients with respect to the Lebesgue measure and the coefficients of $T_{\text {sing }}$ are singular measures. In general, $T_{\text {abc }}$ and $T_{\text {sing }}$ are positive but not closed. We fix an arbitrary set $\Xi \subset X$ and for $p=1,2, \ldots, n, n+1$, we set

$$
b_{p}=b_{p}(T, \Xi)=\inf \left\{c>0 \mid \operatorname{codim}\left(E_{c}(T), x\right) \geq p \text { for all } x \in \Xi\right\}
$$

with the convention that a geam has codimension $>n$ if and only if it is empty. Then $0=b_{1} \leq b_{2} \leq \cdots \leq b_{n} \leq b_{n+1}$ with $b_{n+1}=\max _{x \in \Xi} \nu(T, x)<+\infty$, and for $c \in$ ]$\left.b_{p}, b_{p+1}\right], E_{c}(T)$ has codimension $\geq p$ at every point of $\Xi$ and has at least one component exactly of codimension $p$ which intersects $E$. We call $b_{1}, b_{2}, \ldots$ the 
jumping values of the Lelong numbers of $T$ over $\Xi$.

Let $E \subset X-D$ be an arbitrary subset and $b_{p}=b_{p}(T, E)$. Denote by $\left\{Z_{p, k}\right\}_{k \geq 1}$ the irreducible components of codimension $p$ in $\cup_{c>b_{p}} E_{c}(T)$ which intersect $\Xi$ and let $\left.\nu_{p, k} \in\right] b_{p}, b_{p+1} j$ be the generic Lelong number of $T=c(L) \geq$ 0 along $Z_{p, k}$. With these notations, the following fundamental inequality is a key step of our theory.

Theorem 4. 4. Let $G$ be a semipositive line bundle on $X$. Assume that there is a non negative rational number a such that $Q$-divisor $a G$ is a line bundle in usual sense and such that $\left.(T X \otimes \mathcal{O}(a G))\right|_{X-D}$ is generated by $H^{0}(X, T X \otimes$ $\mathcal{O}(a G))$. Set $u=a c(G) \geq 0$ with any smooth semipositive metric on $G$. Then the de Rham cohomology class $\left(\{T\}+b_{1} u\right) \cdots\left(\{T\}+b_{p} u\right)$ can be represented by a closed positive $(p, p)$-current $\Theta_{p}$ such that

$$
\Theta_{p} \geq \sum_{k \geq 1}\left(\nu_{p, k}-b_{1}\right) \cdots\left(\nu_{p, k}-b_{p}\right)\left[Z_{p, k}\right]+\left(T_{\mathrm{abc}}+b_{1} u\right) \wedge \cdots \wedge\left(T_{\mathrm{abc}}+b_{p} u\right) .
$$

Here $\wedge\left(T_{\mathrm{abc}}+b_{j} u\right)$ is computed pointwise as a $(p, p)$-form. It follows in particular from our inequality that $T_{\mathrm{abc}}^{p}$ has locally integrable coefficients for all $p$.

Proof. By [De 2, Proposition 9. 1], there is an ample line bundle $F$ with the following property : for every $s \in N$ there exist sections $f_{i} \in H^{0}(X, F+s L), 1$ $\leq i \leq N(s)$, with

$$
\nu(T, x)-\frac{n}{s} \leq \frac{1}{s} \min \operatorname{ord}_{x}\left(f_{i}\right) \leq \nu(T, x) \quad \text { for all } x \in X .
$$

The idea is to decrease the Lelong numbers by replacing each section $f_{i}$ by some of its high order derivatives, or rather by some jet section. In this way, the polar components with low generic Lelong number disappear and we can decrease the dimension so as to be able to take intersections of currents (thanks to Proposition 4. 2).

First step : $E_{m}^{*} \otimes \mathcal{O}(3 F+s L+m a G)$ is ample modulo $D$, for $m$-jets bundle $E_{m}=J^{m} \mathcal{O}(F+s L)$.

We show by induction on $m$ that $E_{m-1}^{*} \otimes \mathcal{O}(3 F+s L+m a G)$ satisfies the condition (iii) of Proposition 2.6 for every $m \in N$. For $m=1, E_{0}^{*} \otimes \mathcal{O}(3 F+s L+$ $a G)=2 F+a G$, this is a positive line bundle. There are exact sequences

$$
0 \longrightarrow S^{m} T^{*} X \otimes \mathcal{O}(F+s L) \longrightarrow E_{m} \longrightarrow E_{m-1} \longrightarrow 0
$$

Tensoring $\mathcal{O}(3 F+s L+m a G)$ with the duals, we get the following exact sequences 


$$
\begin{gathered}
0 \longrightarrow E_{m-1}^{*} \otimes \mathcal{O}(3 F+s L+m a G) \longrightarrow E_{m}^{*} \otimes \mathcal{O}(3 F+s L+m a G) \\
\longrightarrow S^{m} T X \otimes \mathcal{O}(2 F+m a G) \longrightarrow 0 .
\end{gathered}
$$

By the induction hypothesis, $E_{m-1}^{*} \otimes \mathcal{O}(3 F+s L+m a G)$ satisfies (iii) of Proposition 2. 6. Since $S^{m} T X \otimes \mathcal{O}(m a G)$ is spanned on $X-D$ by our assumption, $S^{m} T X$ $\otimes \mathcal{O}(F+m a G)$ is ample modulo $D$ by Proposition 6.3 in Section 6 below. By Proposition 2. 9, $S^{m} T X \otimes \mathcal{O}(2 F+m a G)$ satisfies (iv) of Proposition 2.7 and (iii) of Proposition 2. 6 as well. By the proof of Corollary 2. 10, $E_{m}^{*} \otimes \mathcal{O}(3 F+s L+$ $m a G)$ and so $E_{m}^{*} \otimes \mathcal{O}(3 F+s L+(m+1) a G)$ satisfy (iii) of Proposition 2.6. In particular $E_{m}^{*} \otimes \mathcal{O}(3 F+s L+m a G)$ is ample modulo $D$ by Proposition 2. 6 .

Second step : Killing Lelong numbers in the singular metric of $L$.

Consider the $m$-jet section $J^{m} f_{i}$ with values in the vector bundle $E_{m}$ of $m$ jets. By the first step above, $E_{m}^{*} \otimes \mathcal{O}(3 F+s L+m a G)$ is ample modulo $D$. Hence there is a symmetric power of order $q$ such that $S^{q} E_{m}^{*} \otimes \mathcal{O}(3 q F+q s L+q m a G)$ is generated by holomorphic sections $g_{j} \in H^{0}\left(X, S^{q} E_{m}^{*} \otimes \mathcal{O}(3 q F+q s L+q m a G)\right)$ on $X-D$. We use the pairing of $S^{q} E_{m}$ and $S^{q} E_{m}^{*}$ to get sections $S^{q}\left(J^{m} f_{j}\right) \cdot g_{j} \in H^{0}$ $(X, \mathcal{O}(3 q F+q s L+q m a G))$. By means of these sections, we define for each pair $(s, m)$ a new singular metric \|\|$_{s, m}$ on $L$ such that

$$
\|\xi\|_{s, m}=\frac{\|\xi\|}{\sum_{i, j}\left\|S^{q}\left(J^{m} f_{i}\right) \circ g_{j}\right\|^{1 / q s}}, \quad \xi \in \mathbb{L},
$$

where \|\| denotes the original singular metric on $\mathbb{L}$ as well as the induced metric on $\mathcal{O}(3 q F+q s L+q m a G)$, here the metric of $F$ (resp. $G)$ is smooth and has positive (resp. semipositive) curvature. Denote by $\varphi$ the weight of the original metric on $L$, by $\varphi_{s, m}$ the new one, and by $\psi_{F}, \psi_{G}$ on some trivializing open set $U \subset X$. Then

$$
\varphi_{s, m}=\frac{1}{q S} \log \sum_{l, J}\left|S^{q}\left(J^{m} f_{i}\right) \circ g_{j}\right|-\frac{3}{s} \varphi_{F}-\frac{m}{s} a \varphi_{G},
$$

because $e^{-\varphi}$ appears in the numerator and exp $\left(-3 q \varphi_{F}-q s L-q m a \varphi_{G}\right)^{\frac{1}{q s}}$ in the denominator of $\|\xi\|_{s, m}$. As $\varphi_{F}, \varphi_{G}$ are smooth and the $g_{j}$ 's do not vanish simultaneusly on $X-D$, we get

$$
\nu\left(\varphi_{s, m}, x\right)=\frac{1}{s} \min _{i} \operatorname{ord}_{x}\left(J^{m} f_{i}\right)=\frac{1}{s}\left(\min _{i} \operatorname{ord}_{x}\left(f_{i}\right)-m\right)+\text { for } x \in X-D,
$$

where $t_{+}:=\max \{t, 0\}$ for $t \in \boldsymbol{R}$. Hence we have inequality

$$
\left(\nu(T, x)-\frac{m+n}{s}\right)_{+} \leq \nu\left(\varphi_{s, m}, x\right) \leq\left(\nu(T, x)-\frac{m}{s}\right)_{+} \text {for } x \in X-D,
$$


that is, we have been able to construct a new curvature current $\frac{\sqrt{-1}}{\pi} \partial \bar{\partial} \psi_{s, m}$ on $L$ in which all the Lelong numbers that were $\leq \frac{m}{s}$ have been killed on $X-D$. Unfortunately the curvature is no longer $\geq 0$, but by (4.5) we have

$$
\frac{\sqrt{-1}}{\pi} \partial \bar{\partial} \psi_{s, m} \geq-\frac{3}{s} c(F)-\frac{m}{s} a c(G)=-\frac{3}{s} \omega-\frac{m}{s} u \text { on } X-D,
$$

where $\omega=c(F)>0$. Only the term $\frac{3}{s} \omega$ can be made arbitrarily small. For each $s$, select an integer $m$ such that $b_{p}<\frac{m}{s}<b_{p}+\frac{1}{s}$. By (4. 5) and (4.6), we see that $\varphi_{s, m}$ is locally bounded on $X-D-E_{m / s}(T)$ and the definition of $b_{p}$ implies that $E_{m / s}(T)$ has codimension $\geq p$ in a neighborhood of $\Xi$.

Third step : Construction of the $p$ th intersection current $\Theta_{p}$.

By induction on $p$, we suppose that $\Theta_{p-1}$ has been constructed $\left(\Theta_{1}=T\right.$ satisfies the requirements for $p=1$ ). By Proposition 4.2, the wedge product $\Theta_{p-1} \wedge \frac{\sqrt{-1}}{\pi} \partial \bar{\partial} \psi_{s, m}$ is well defined in a neighborhood of $\Xi$. Since $L$ is nef, there is a smooth metric on $L$ for each $s$, associated with some weight $\rho_{s}$ on the trivializing open set $U$, such that $\frac{\sqrt{-1}}{\pi} \partial \partial \rho_{s} \geq-\frac{1}{s} \omega$. We introduce the weight

$$
\phi_{s, m, A, B}=\sup \left(\varphi, \varphi_{s, m}-A, \rho_{s}-B\right)
$$

where $A, B>0$ are large constants. This weight corresponds to the singular metric on $L$ given by

$$
\|\xi\|_{s, m, A, B}=\inf \left(\|\xi\|, e^{A}\|\xi\|_{s, m}, e^{B}\|\xi\|_{\rho_{s}}\right) .
$$

Clearly $\phi_{s, m, A, B}$ converges to $\varphi$ as $A, B$ tend to $+\infty$ and $\phi_{s, m, A, B}$ is locally bounded, therefore the curvature current $T_{s, m, A, B}=\frac{\sqrt{-1}}{\pi} \partial \bar{\partial} \phi_{s, m, A, B}$ converges weakly to $T=\frac{\sqrt{-1}}{\pi} \partial \bar{\partial} \varphi$ as $A, B$ tend to $+\infty$. Moreover, the assumed lower bound on $\frac{\sqrt{-1}}{\pi} \partial \bar{\partial} \rho_{s}$ combined with (4.7) implies

$$
T_{s, m, A, B} \geq-\frac{3}{s} \omega-\frac{m}{s} u \text { on } X-D,
$$

this is seen by adding $\frac{3}{s} \psi_{F}+\frac{m}{s} a \psi_{G}$ to each of the three terms in the supremum formula for $\psi_{s, m, A, B}$. The positive $(p, p)$-current

$$
\Theta_{p, s, m, A, B}=\Theta_{p-1} \wedge\left(T_{s, m, A, B}+\frac{3}{s} \omega+\frac{m}{s} u\right)
$$

is well defined over $X-D$ and well defined as a current on $X$, since $\phi_{s, m, A, B}$ is locally bounded on $X-D$ and since the subset $D$ of $X$ is of measure zero. Its cohomology class is independent of $A, B$ and converges to $\left\{\Theta_{p-1}\right\} \cdot\left(c_{1}(L)+\right.$ $b_{p} u$ ) when $s$ tends to $+\infty$ (by the choice of $m$ made at the end of the second step, we have $\left.\lim m / s=b_{p}\right)$. Hence the family $\left(\Theta_{p, s, m, A, B}\right)$ is weakly compact. First extract a weak limit $\Theta_{p, s, m, A}$ by taking some subsequence $\boldsymbol{B}_{\nu} \rightarrow+\infty$. By 
Proposition 4. 2, we see that in a neighborhood of $E$

$$
\Theta_{p, s, m, A}:=\lim _{\mathbf{B} \rightarrow+\infty} \Theta_{p, s, m, A, B}=\Theta_{p-1} \wedge\left(T_{s, m, A}+\frac{3}{s} \omega+\frac{m}{s} u\right),
$$

where $T_{s, m, A}=\frac{\sqrt{-1}}{\pi} \partial \bar{\partial} \psi_{s, m, A}, \phi_{s, m, A}=\sup \left(\varphi, \varphi_{s, m}-A\right)$. Indeed the codimension of the set of poles of $\phi_{s, m, A}$ is at least $p$ in a neighborhood of $\Xi$. By (4.6), we have

$$
\nu\left(\phi_{s, m, A}, x\right) \geq \min \left(\nu(\varphi, \mathrm{x}), \nu\left(\varphi_{s, m}, x\right)\right) \geq\left(\nu(T, x)-\frac{m+n}{s}\right)_{+} .
$$

Proposition 4. 2 shows that

$$
\nu\left(\Theta_{p, s, m, A}, x\right) \geq \nu\left(\Theta_{p^{-1}}, x\right)\left(\nu(T, x)-\frac{m+n}{s}\right)_{+} \text {near } \Xi
$$

By induction on $p$, we conclude that the generic Lelong number of $\Theta_{p, s, m, A}$ along $Z_{p, k}$ is at least equal to

$$
\left(\nu_{p, k}-b_{1}\right) \cdots\left(\nu_{p, k}-b_{p-1}\right)\left(\nu(T, x)-\frac{m+n}{s}\right)_{+} .
$$

In fact, $Z_{p, k}$ meets $\Xi$ at some point $x$, and therefor the inequality hold at least on a neighborhood of $x$ in $Z_{p, k}$. Siu's decomposition formula (3.1) yields

$$
\Theta_{p, s, m, A} \geq\left(\nu_{p, k}-b_{1}\right) \cdots\left(\nu_{p, k}-b_{p-1}\right)\left(\nu(T, x)-\frac{m+n}{s}\right)_{+}\left[Z_{p, k}\right] .
$$

Take a weak limit $\Theta_{p, s, m}$ for some subsequence $A_{\nu} \rightarrow+\infty$ and then a weak limit $\Theta_{p}$ for some subsequence $m_{\nu} / s_{\nu} \rightarrow b_{p}$ with $s_{\nu} \rightarrow+\infty$. We obtain a current $\Theta_{p}$ such that $\left\{\Theta_{p}\right\}=\left\{\Theta_{p-1}\right\} \bullet\left(c_{1}(L)+b_{p}\{u\}\right)$ and

$$
\Theta_{p} \geq\left(\nu_{p, k}-b_{1}\right) \cdots\left(\nu_{p, k}-b_{p}\right)\left[Z_{p, k}\right]
$$

It remains only to show by induction on $p$ that

$$
\Theta_{p, \mathrm{abc}} \geq\left(T_{\mathrm{abc}}+b_{1} u\right) \wedge \cdots \wedge\left(T_{\mathrm{abc}}+b_{p} u\right)
$$

As the coefficients of $\left[Z_{p, k}\right]$ are singular with respect to the Lebesgue measure, $\Theta_{p}$ will actually be larger than the sum. By construction, there exists a subsequence $\left(s_{\nu}, m_{\nu}, A_{\nu}, B_{\nu}\right)$ such that

$$
\begin{gathered}
\Theta_{p}=\lim \Theta_{p-1} \wedge\left(\frac{\sqrt{-1}}{\pi} \partial \bar{\partial} \varphi_{s_{\nu}, m_{\nu}, A_{\nu}, B_{\nu}}+\frac{3}{s_{\nu}} \omega+\frac{m_{\nu}}{s_{\nu}} u\right), \\
\phi_{s_{\nu}, m_{\nu}, A_{\nu}, B_{\nu}}=\sup \left(\varphi, \varphi_{s_{\nu}, m_{\nu}}-A_{\nu}, \rho_{s_{\nu}}-B_{\nu}\right) .
\end{gathered}
$$


The desired lower bound follows from Lemma 4.8 below.

Lemma 4.8 ([De 2, Lemma 10.12]). Let $U \subset C^{n}$ be an open subset and let $\varphi$ be an arbitrary plurisubharmonic function on $U$. Let $\varphi_{\nu}=\max \left(\varphi, \phi_{\nu}\right)$ where $\phi_{\nu}$ is a decreasing sequence of plurisubharmonic functions converging to $-\infty$, each $\phi_{\nu}$ being locally bounded in $U$. Let $\Theta$ be a closed positive $(p-1, p-1)$-current. If $\Theta \wedge \sqrt{-1} \partial \bar{\partial} \varphi_{\nu}$ converges to a weak limit $\Theta^{\prime}$, then

$$
\Theta_{\mathrm{abc}}^{\prime} \geq \Theta_{\mathrm{abc}} \wedge(\sqrt{-1} \partial \bar{\partial} \varphi)_{\mathrm{abc}}
$$

We give a sufficient condition for (3.12). Frist suppose that $L$ is an ample line bundle over $X$. The idea is to apply the inequalty 4.4 to the $(1,1)$-current $T=\lim \omega_{\varepsilon_{\nu}}$, the limit of solutions of the equation (3.7), and to integrate the inequalty with respect to the Kähler form $\omega=c(L)$. Before doing this, we need some convex inequalities and have to estimate the excess of intersection in terms of $T_{\mathrm{abc}}^{n}$.

Proposition 4.9 ([De 2, Proposition 11.1]). The absolutely continuous part $T_{\mathrm{abc}}$ of $T$ satisfies

$$
T_{\mathrm{abc}}^{n} \geq\left(1-\frac{\sigma}{L^{n}}\right) \omega^{n} \text { a. e. on } X .
$$

Proposition 4. 10 ([De 2, Proposition 5. 2]). In any dimension $n$ :

(a) if $\alpha_{1}, \ldots, \alpha_{n}$ are semipositive $(1,1)$-forms on $C^{n}$, then

$$
\alpha_{1} \wedge \alpha_{2} \wedge \cdots \wedge \alpha_{n} \geq\left(\alpha_{1}^{n}\right)^{1 / n}\left(\alpha_{2}^{n}\right)^{1 / n} \cdots\left(\alpha_{n}^{n}\right)^{1 / n}
$$

(b) if $u_{1}, \cdots, u_{n}$ are semipositive cohomology classes of type $(1,1)$ on $X$, then

$$
u_{1} \cdot u_{2} \cdots u_{n} \geq\left(u_{1}^{n}\right)^{1 / n}\left(u_{2}^{n}\right)^{1 / n} \cdots\left(u_{n}^{n}\right)^{1 / n}
$$

We consider an arbitrary subset $\Xi \subset X-D$ and $b_{p}=b_{p}(T, \Xi)$. By Proposition 4.9 and the inequality $4.10(\mathrm{a})$, we have

$$
T_{\mathrm{abc}}^{j} \wedge \omega^{n-j} \geq\left(1-\frac{\sigma}{L^{n}}\right)^{\frac{J}{n}} \omega^{n}
$$

Suppose that $T X \otimes a L$ is spanned on $X-D$ for some $a \geq 0$ as in Theorem 4. 4. We can then apply Theorem 4.4 with $u=a \omega$ and $\left\{\Theta_{p}\right\}=\left(1+b_{1} a\right) \cdots\left(1+b_{p} a\right)$ $\left\{\omega^{p}\right\}$. By taking the wedge product of $\Theta_{p}$ with $\omega^{n-p}$, we get 


$$
\begin{aligned}
\left(1+b_{1} a\right) \cdots\left(1+b_{p} a\right) \int_{X} \omega^{n} \geq & \sum_{k \geq 1}\left(\nu_{p, k}-b_{1}\right) \cdots\left(\nu_{p, k}-b_{p}\right) \int_{X}\left[\mathbb{Z}_{p, k}\right] \wedge \omega^{n-p} \\
& +\int_{X}\left(T_{\mathrm{abc}}+b_{1} a \omega\right) \wedge \cdots \wedge\left(\mathbb{T}_{\mathrm{abc}}+b_{p} a \omega\right) \wedge \omega^{n-p} .
\end{aligned}
$$

Combining this inequalty with (4.11) for $T_{\mathrm{abc}}^{p-j}$, we find

$$
\begin{array}{r}
\left(1+b_{1} a\right) \cdots\left(1+b_{p} a\right) L^{n} \geq \sum_{k \geq 1}\left(\nu_{p, k}-b_{1}\right) \cdots\left(\nu_{p, k}-b_{p}\right) \mathbb{L}^{n-p} \circ \mathbb{Z}_{p, k} \\
+\sum_{0 \leq j \leq p} S_{j}^{p}(b) a^{j}\left(1-\frac{\sigma}{\mathbb{L}^{n}}\right)^{(p-j) / n} \mathbb{L}^{n},
\end{array}
$$

where $S_{j}^{p}(b), 1 \leq j \leq p$, denotes the elementary symmetric polynomial of degree $j$ in $b_{1}, \ldots, b_{p}$ and $S_{0}^{p}(b)=1$. Since $\Pi\left(1+b_{j} a\right)=\sum S_{j}^{p}(b) a^{j}$, we get

$$
\sum_{k \geq 1}\left(\nu_{p, k}-b_{1}\right) \cdots\left(\nu_{p, k}-b_{p}\right) L^{n-p} \cdot \mathbb{Z}_{p, k} \leq \sum_{0 \leq j \leq p} S_{j}^{p}(b) a^{J}\left(1-\left(1-\frac{\sigma}{\mathbb{L}^{n}}\right)^{(p-j) / n}\right) L^{n}
$$

If $L$ is only supposed to be nef and ample modulo $D$, we follow essentially the same arguments and replace $\omega$ in all our inequalities by $\omega_{m}=c(\mathbb{L})_{m}+\frac{1}{m} c(A)$ with an ample line bundle $A$ (see below of Corollary 3.10). Note that all $(n, n)-$ forms $\omega_{m}^{n}$ were defined to be proportional to $\gamma^{n}=c(A)^{n}$, so the inequalty 4.9 becomes in the limit

$$
T_{\mathrm{abc}}^{n} \geq\left(1-\frac{\sigma}{L^{n}}\right) \frac{L^{n}}{A^{n}} \gamma^{n}=\left(1-\frac{\sigma}{L^{n}}\right) \frac{L^{n}}{\left(L+\frac{1}{m} A\right)^{n}} \omega_{m}^{n}
$$

In the inequality (4. 12), $\nu_{p, k}$ is the generic Lelong number of $T$ along $\mathbb{Z}_{p, k}$ and $\mathbb{Z}_{p, k}$ runs over all $p$-codimensional components $Y$ of $\cup_{c>b} E_{c}(T)$ intersecting $E$. By definition of $b_{j}$ we have $\max _{k} \nu_{p, k}=b_{p+1}$. Hence we obtain :

Proposition 4. 13. Let $\mathbb{L}$ be a nef line bundle on $X$. Assume that $L$ is ample modulo $D$ and that $T X \otimes \mathcal{O}(a L)$ is spanned on $X-D$ for some $a \geq 0$ as in Theorem 4. 4. Let $T \in c_{1}(L)$ be the positive curvature current obtained by the limit of solutions of the equations (3.7) and (3.11). Then $b_{p}=b_{p}(T, \Xi)$ for an arbitrary subset $\Xi \subset X-D$ satisfy the inductive inequalities

$$
\left(b_{p+1}-b_{1}\right) \cdots\left(b_{p+1}-b_{p}\right) \leq \frac{1}{\min _{Y} L^{n-p} \circ Y_{0 \leq j \leq p-1}} \sum_{j}(b) a^{j} \sigma_{p-j},
$$

where $\sigma_{j}=\left(1-\left(1-\frac{\sigma}{L^{n}}\right)^{\frac{j}{n}}\right) L^{n}$ and where $Y$ runs over all $p$-codimensional subvarieties of $X$ intersecting $\Xi$. 
Observe that $\sigma_{j}$ is increasing in $j$, in paticular $\sigma_{j}<\sigma_{n}=\sigma$ for $j \leq n-1$. Moreover, the convexity of the exponential function shows that $t \mapsto \frac{1}{t}\left(1-\left(1-\frac{\sigma}{L^{n}}\right)^{t}\right)$ $L^{n}$ is decreasing, thus $\sigma_{j}>\sigma_{p} j / p$ for $j<p$, in particular $\sigma_{j}>\sigma j / n$ for $j \leq n-1$. We are now in a position to prove the following general result. Let $g_{j}:\left(X, x_{j}\right) \rightarrow$ $\left(C^{n}, 0\right)$ be germs of finite holomorphic maps with covering degree $\rho_{j}$ and with $x_{j}$ $\in X-D$. Let $\mathscr{J}_{1} \subset \mathcal{O}_{X, x_{1}}, \ldots, \mathscr{J}_{N} \subset \mathcal{O}_{X, x_{N}}$ be the associated ideals $\mathscr{I}\left(\tau_{j, 0} \log \right.$ $\left.\left|g_{j}\right|\right)_{x_{j}}$ and let

$$
\sigma_{0}=\sum \rho_{j} \tau_{j, 0}^{n}, \quad \sigma_{p}=\left(1-\left(1-\frac{\sigma_{0}}{L^{n}}\right)^{p / n}\right) L^{n}, 1 \leq p \leq n-1
$$

With these symbols in mind, we have :

Theorem 4.15. Let $L$ be a nef line bundle on $X$. Assume that $L$ is ample modulo $D$ and that $T X \otimes \mathcal{O}(a L)$ is spanned on $X-D$ for some $a \geq 0$ as in Theorem 4. 4 and that there is a sequence $0=\beta_{1}<\cdots<\beta_{n} \leq 1$ with

$$
L^{n-p} \cdot Y>\left(\beta_{p+1}-\beta_{1}\right)^{-1} \cdots\left(\beta_{p+1}-\beta_{p}\right)^{-1} \sum_{0 \leq j \leq p-1} S_{j}^{p}(\beta) a^{j} \sigma_{p-j}
$$

for every subvariety $Y \subset X$ of codimension $p=1,2, \ldots, n-1$ passing through one of the points $x_{j}$. Then there is a surjective map

$$
H^{0}\left(X, K_{X}+L\right) \rightarrow \bigoplus_{1 \leq J \leq N} \mathcal{O}\left(K_{X}+L\right)_{x_{j}} \otimes\left(\mathcal{O}_{X, x_{j}} / \mathscr{J}_{j}\right) .
$$

Proof. Select $\tau_{j}>\tau_{j, 0}$ so that $L^{n-p} \bullet Y$ still satisfies the above lower bound with the corresponding value $\sigma>\sigma_{0}$. Then apply Proposition 4.13 with $E=\left\{x_{1}\right.$, $\left.\ldots, x_{N}\right\}$. Inequality (4.14) shows inductively that $b_{p}<\beta_{p}$ for $p \geq 2$, so $b_{n}<1$ and we get codim $\left(E_{1}(T), x_{j}\right)=n$ at each point $x_{j}$. Thanks to (3.2), Corollary 3. 5 and Corollary 3. 10 imply the desired surjectivity property.

\section{$\S 5$. Very Ampleness Criterion on Open Varieties}

In this section, we will prove Theorem 1 and Theorem 2. Throughout of this section, we assume that $X$ is an $n$-dimensional projective manifold, that $D$ is a reduced effective divisor on $X$ with only simple normal crossings and that $K_{X}+$ $D$ is nef.

Let $L$ be a nef line bundle on $X$ and assume that $L$ is ample modulo $D$. To use the results of section 4 , we have to find some constant $a \geq 0$ such that $T X \otimes$ $a L$ is spanned on $X-D$.

Lemma 5.1. Let $F$ be a nef line bundle over $X$. Assume that $F$ is very ample modulo D. Then $T X \otimes \mathcal{O}\left(K_{X}+D+n F\right)$ is generated by $H^{0}\left(X, T X \otimes \mathcal{O}\left(K_{X}+D+\right.\right.$ 
$n F)$ ) over $X-D$.

Proof. Since $F$ is very ample modulo $D$, the 1 -jet bundle $J^{1} F$ is generated by global sections over $X-D$. Consider the exact sequence

$$
0 \longrightarrow T X^{*} \otimes F \longrightarrow J^{1} F \longrightarrow F \longrightarrow 0
$$

where $\operatorname{rank}\left(J^{1} F\right)=n+1$ and $\operatorname{det}\left(J^{1} F\right)=K_{X}+(n+1) F$. The $n$-th extrior power $\wedge^{n}\left(J^{1} F\right)$ is also generated by global sections over $X-D$ and there is a surjective morphism

$$
\bigwedge^{n}\left(J^{1} F\right)=\left(J^{1} F\right)^{*} \otimes \operatorname{det}\left(J^{1} F\right) \longrightarrow\left(T X \otimes F^{*}\right) \otimes \operatorname{det}\left(J^{1} F\right)=T X \otimes \mathcal{O}\left(K_{X}+n F\right) .
$$

Hence $T X \otimes \mathcal{O}\left(K_{X}+n F\right)$, and so $T X \otimes \mathcal{O}\left(K_{X}+D+n F\right)$ is generated by global sections over $X-D$.

The next idea based on the following iteration trick ([De 2, Lemma 12. 2]) : Lemma 5.1 suggests that a universal lower bound for the spannedness on $X-D$ of $T X \otimes \mathcal{O}\left(a L^{\prime}\right)$ can be achieved with $L^{\prime}=K_{X}+D+L$ if $L$ is sufficiently ample modulo $D$. Then it follows by Theorem 4.15 that $K_{X}+D+L^{\prime}=2\left(K_{X}+\right.$ $D)+L$ is nef and very ample modulo $D$ under suitable numerical conditions. Lemma 5. 1 applied with $F=2\left(K_{X}+D\right)+L$ shows that $T X \otimes \mathcal{O}\left((2 n+1)\left(K_{X}+\right.\right.$ $D)+n L)$ is generated by global sections over $X-D$, and thus $T X \otimes \mathcal{O}((2 n+1)$ $\left.L^{\prime \prime}\right)$ is spanned on $X-D$ with $L^{\prime \prime}=K_{X}+D+\frac{1}{2} L \leq L$ ! Hence we see that Theorem 4.15 can be iterated.

Lemma 5. 2. Let $L^{\prime}$ be a nef line bundle over $X$ which is ample modulo $D$. Assume that $T X \otimes \mathcal{O}\left((2 n+1) L^{\prime}\right)$ is spanned on $X-D$. Then $K_{X}+D+L^{\prime}$ is very ample modulo $D$, insofar as

$$
\mu_{X-D}\left(L^{\prime}\right):=\min _{1 \leq p \leq n} \min _{\substack{\operatorname{dim} Y=p \\ Y \cap(X-D) \neq 0}}\left(L^{\prime p} \cdot Y\right)^{1 / p}>2 C_{n} n^{n}
$$

with a positive constant $C_{n}<3$ depending only on $n$.

Proof. This is only a matter of straightforward calculations as in [De 2, Corollary 1]. Hence we shall give only the outline of the proof. Let $\mu=\mu_{X-D}$ $\left(L^{\prime}\right), a=2 \mathrm{n}+1$ and $\sigma_{0}=2 n^{n}$. By the convexity argument already explained, we have $\sigma_{p-j} \leq(p-j) \sigma_{1} \leq p\left(1-\frac{1}{p}\right)^{j} \sigma_{1}$. As $\beta_{1}=0$, we find

$$
\sum_{0 \leq j \leq p-1} S_{j}^{p}(\beta) a^{j} \sigma_{p-j} \leq p \sigma_{1} \sum_{0 \leq j \leq p-1} S_{j}^{p}(\beta)\left(\frac{p-1}{p} a\right)^{j}
$$




$$
=p \sigma_{1}\left(1+\beta_{2} \frac{p-1}{p} a\right) \cdots\left(1+\beta_{p} \frac{p-1}{p} a\right) .
$$

When we replace $L^{\prime}$ by $m L^{\prime}$, the constant $a$ is replaced by $\frac{a}{m}$, and by definition of $\mu=\mu_{X-D}\left(L^{\prime}\right)$ we have $\left(m L^{\prime}\right)^{k} \cdot Y \geq(m \mu)^{k}$. Hence Theorem 4. 15 yields the sufficient condition

$$
(m \mu)^{n-p}>\prod_{j \leq p}\left(\beta_{p+1}-\beta_{j}\right)^{-1} p \sigma_{1}\left(1+\beta_{2} \frac{p-1}{p} \frac{a}{m}\right) \cdots\left(1+\beta_{p} \frac{p-1}{p} \frac{a}{m}\right)
$$

with $0=\beta_{1}<\cdots<\beta_{n}<1$. We suppose $\mu \geq \lambda \sigma_{0}$ and choose

$$
\beta_{p}=\left(\alpha^{n-p+1}\left(2 n^{n}\right)^{n-p}\right)^{\frac{1}{p-1}}, 2 \leq p \leq n-1
$$

with suitable constants $\lambda, \alpha$ to be determined later. If we set the constants

$$
\begin{gathered}
U_{p}^{\prime}=\prod_{2 \leq J \leq p}\left(1-\frac{\beta_{j}}{\beta_{p+1}}\right)^{-1}, \quad V_{p}^{\prime}=\prod_{2 \leq j \leq p}\left(1+\frac{p-1}{p} \beta_{j}(2 n+1)\right), \\
T_{n}^{\prime}=\left(1-\left(1-\lambda^{-n}\left(2 n^{n}\right)^{-(n-1)}\right)^{1 / n}\right) \lambda^{n}\left(2 n^{n}\right)^{n-1} .
\end{gathered}
$$

We have $\sigma_{1} \leq T_{n}^{\prime} \sigma_{0}$ and our conditions become

$$
\mu^{n-p}>U_{p}^{\prime} \beta_{p+1}^{p} p T_{n}^{\prime} \sigma_{0} V_{p}^{\prime}= \begin{cases}p U_{p}^{\prime} V_{p}^{\prime} T_{n}^{\prime} \alpha^{n-p}\left(2 n^{n}\right)^{n-p-1} \sigma_{0} & \text { for } p \leq n-2, \\ (n-1) U_{n-1}^{\prime} V_{n-1}^{\prime} T_{n}^{\prime} \sigma_{0} & \text { for } p=n-1 .\end{cases}
$$

A sufficient condition is

$$
\mu>\sigma_{0} \max \left\{(n-1) U_{n-1}^{\prime} V_{n-1}^{\prime} T_{n}^{\prime}, \alpha\left(p U_{p}^{\prime} V_{p}^{\prime} T_{n}^{\prime}\right)^{1 /(n-p)}\right\}_{1 \leq p \leq n-2}
$$

We adjust $\lambda$ and $\alpha$ so that

$$
(n-1) U_{n-1}^{\prime} V_{n-1}^{\prime} T_{n}^{\prime}=\alpha \max _{1 \leq p \leq n-2}\left(p U_{p}^{\prime} V_{p}^{\prime} T_{n}^{\prime}\right)^{1 /(n-p)}=\lambda,
$$

and we take this common value to be our constant $C_{n}$. A numerical caluculation gives $C_{n}<3$ for all $n$.

Proof of Theorem 1. Since $L$ is nef and ample modulo $D$, there exists an integer $q$ such that

$$
\begin{cases}K_{X}+D+q L & \text { is nef and ample modulo } D, \\ T X \otimes \mathcal{O}\left((2 n+1)\left(K_{X}+D+q L\right)\right) & \text { is spanned on } X-D, \\ \mu_{X-D}\left(K_{X}+D+q L\right)>2 C_{n} n^{n}, & \end{cases}
$$

where $C_{n}$ is a constant in Lemma 5. 2. By Lemma 5. 1 applied to $L^{\prime}=K_{X}+D+$ 
$q L$, we find that $F=K_{X}+D+L^{\prime}=2\left(K_{X}+D\right)+q L$ is nef and very ample modulo $D$. In particular, $K_{X}+D+\frac{q}{2} L$ is, as a $Q$-divisor, nef and ample modulo $D$. Hence, for any subvariety $Y \subset X$ of codimension $p$ not contained in $D$, we have

$$
\begin{aligned}
\left(K_{X}+D+(q-1) \mathbb{L}\right)^{p} \circ Y & =\left(\frac{1}{2} F+\left(\frac{q}{2}-1\right) L\right)^{p} \circ \mathbb{Y} \\
& =\sum_{0 \leq k \leq p}{ }_{p} C_{k} 2^{k-p}\left(\frac{q}{2}-1\right)^{k} \mathbb{F}^{p-k} \circ L^{k} \circ Y .
\end{aligned}
$$

By the convexity inequality 4.10 (b) and we get

$$
F^{p-k} \circ L^{k} \circ Y \geq\left(F^{p} \cdot Y\right)^{1-\frac{k}{p}}\left(L^{p} \circ Y\right)^{\frac{k}{p}} \geq\left(\mu_{X-D}(L)\right)^{k}
$$

Hence $\left(K_{X}+D+(q-1) L\right)^{p} \circ Y \geq\left(\left(\frac{q}{2}-1\right) \mu_{X-D}(L)+\frac{1}{2}\right)^{p}$ and

$$
\mu_{X-D}\left(K_{X}+D+(q-1) L\right) \geq \frac{1}{2}\left((q-2) \mu_{X-D}(\mathbb{L})+1\right) .
$$

Moreover, Lemma 5. 1 applied to $F$ shows that

$$
T X \otimes \mathcal{O}\left(K_{X}+D+n F\right)=T X \otimes \mathcal{O}\left((2 n+1)\left(K_{X}+D\right)+n q L\right)
$$

is spanned on $X-D$. Since $n q /(2 n+1) \leq q / 2 \leq q-1$ for $q \geq 2$, we have :

$$
\begin{cases}K_{X}+D+(q-1) L & \text { is nef and ample modulo } D \\ T X \otimes \mathcal{O}\left((2 n+1)\left(K_{X}+D+(q-1) L\right)\right) & \text { is spanned on } X-D \\ \mu_{X-D}\left(K_{X}+D+(q-1) L\right) \geq \frac{1}{2}\left((q-2) \mu_{X-D}(L)+1\right) . & \end{cases}
$$

By induction we conclude that (5.4) is still true for the smallest integer $q-1=$ $m$ such that

$$
(q-2) \mu_{X-D}(L)+1=(m-1) \mu_{X-D}(L)+1>4 C_{n} n^{n} .
$$

For this value of $m$, Lemma 5.2 shows that $2\left(K_{X}+\mathbb{D}\right)+m L$ is very ample modulo $D$.

Proof of Theorem 2. If $K_{X}+D$ is nef and ample modulo $D$, the assertion is a direct corollary of Theorem 1. Assume that $-\left(K_{X}+D\right)$ is nef and ample modulo $D$. In the proof of Theorem 1 , we assume that $K_{X}+D$ is nef only to use the iteration trick and to show that $K_{X}+D+L$ is again nef for a nef line bundle $L$. So we can apply our argument for $\mathbb{L}=-m\left(K_{X}+D\right)$ with appropriate $m>0$. 


\section{§ 6. Appendix}

In this section, we shall mention of some elementary properties of vector bundles which are ample modulo $D$. The proof is the same as in [Ha 1, §2]. Let $X$ be a complex projective manifold, $D$ be a divisor with only simple normal crossings, and let $E, E_{1}$ and $E_{2}$ be vector bundles on $X$ (the following propositions even hold for vector bundles on a scheme $X$ of finite type over an algebraically closed field $k$ and non trivial (Zariski) closed subset $D$ ).

Proposition 6. 1. In order for $E$ to be ample modulo $D$, it is sufficient that for every torsion free sheaf $\mathscr{F}$, and for every closed point $x \in X-D$, there be an integer $n_{0}>0$ such that for every $n \geq n_{0}$ the global sections of the sheaf $\mathscr{F} \otimes S^{n}(E)$ generate its stalk at the point $x$, as a modulo over the local ring at that point.

Proposition 6.2. Any quotient of an ample modulo $D$ bundle is ample modulo $D$. $\quad E_{1} \oplus E_{2}$ is ample modulo $D$ if and only if $E_{1}$ and $E_{2}$ are both ample modulo $D$.

Corollary 6. 3. If $E_{1}$ is ample modulo $D$, and $E_{2}$ is spanned on $X-D$, then $E_{1} \otimes E_{2}$ is ample modulo $D$.

Proposition 6. 4. If $E$ is ample modulo $D$, then $S^{n}(E)$ is ample modulo $D$ for all large enough $n$. Conversery, if $S^{n}(E)$ is ample modulo $D$ for some $n$, then $E$ is ample modulo $D$.

Corollary 6.5. Let $L$ be an ample modulo $D$ line bundle. Then $E$ is ample modulo $D$ if and only if $L^{*} \otimes S^{n}(E)$ is spanned on $X-D$ for some $n>0$.

Corollary 6. 6. If $E$ is ample modulo $D$ of rank $r$, then its highest exterior power $\wedge^{r} E$ is also ample modulo $D$.

\section{References}

[B-T 1] Bedford, E. and Taylor, B. A., The Dirichlet problem for the complex Monge-Ampère equation, Invent. Math., 37 (1976), 1-44.

[B-T 2] — A new capacity for plurisubharmonic functions, Acta Math., 149 (1982), 141.

[De 1] Demailly, J-P., Estimation $L^{2}$ pour l'opérateur $\partial$ d'un fibré vectoriel holomorphe semipositif au dessus d'une variété Kählérienne complète, Ann. Sci, Ecole Norm. Sup., 115 (1982), 457-511.

[De 2] - A numerical criterion for very ample line bundles, J. Differntial Geometry, 37 (1993), 323-374. 
[G-H] Griffiths, P. and Harris, J., Principles of Algebraic Geometry, John Wiley and Sons, New York, 1978.

[Ha 1] Hartshorn, R., Ample vector bundles, Inst. Haut. Etud. Sci., 29 (1965), 63-94.

[Ha 2] — Algebraic Geometry, Springer-Verlag, Heidelberg, GTM 52, 1977.

[Hö] Hörmander, L., An introduction to Complex Analysis in several variables, North-Holland Math. Libr., 1966.

[Ii] Iitaka, S., On D-dimensions of algebraic varieties, J. Math. Soc. Japan, 23 (1971), 356 $-373$.

[Ka] Kawamata, Y., A generalization of Kodaira-Ramanujiam's vanishing theorem, Math. Ann., 261 (1982), 43-46.

[Ki] Kiselman, C. O., Sur la dèfinition de l'opérateur de Monge-Ampère complex, Springer Lecture Notes in Math., 1094 (1984), 139-150.

[Le] Lelong, P., Integration sur un ensemble analytique complexe, Bull. Soc. Math. France, 85 (1957), 239-262.

[Na ] Nadel, A. M., Multiplier ideal sheaves and existence of Kähler-Einstein metrics of positive scalar curvature, Ann. of Math., 132 (1990), 549-596.

[No] Norimatsu, Y., Kodaira Vanishing Theorem and Chern Classes for $\partial$-Manifolds, Proc. Japan Acad., 54 (1978), 107-108.

[Oh] Ohsawa, T., Vanishing Theorems on complete Kähler manifolds, Publ. RIMS Kyoto Univ., 20 (1984), 21-38.

[Sa] Sakai, F., Semi-stable curves on algebraic surfaces and logarithmic pluricanonical maps, Math. Ann., 254 (1980), 89-120.

[Si] Siu, Y.-T., Analyticity of sets associated to Lelong numbers and the extension of closed positive current, Invent. Math., 27 (1974), 53-156.

[Vi] Viehweg, E., Vanishing theorems, J. Reine Angew. Math., 335 (1982), 1-8.

[Ya] Yau, S. T., On the Ricci curvature of a compact Kähler manifold and the complex Monge-Ampère equation I, Comm. Pure Appl. Math., 311 (1978), 339-411.

$\left[\mathrm{Zu}_{\lrcorner}\right.$Zucker, S., Hodge Theory with degenerating coefficients, Ann. of Math., 109 (1979), 415-476. 